\title{
THE EXPANSION OF CONTINUOUS FUNCTIONS IN SERIES OF INTEGRALS OF ORTHONORMAL FUNCTIONS
}

\author{
by H. C. FINLAYSON
}

(Received 6 February, 1970; revised 22 October, 1970)

Introduction. This paper deals with the following problem: Can an arbitrary continuous function on $[0,1]$, which vanishes at the origin, be represented in some sense as a series of constant multiples of indefinite integrals of a complete orthonormal set of functions on $[0,1]$ ? Four contexts in which this problem arises naturally will be given in the introduction and the remainder of the paper will be devoted to giving a partial answer to the specific problem formulated in one of these contexts.

Some notation will first be needed. Let $C$ be the space of continuous functions $x(s)$ on $[0,1]$ such that $x(0)=0$. Let $\left\{\alpha_{i}(s)\right\}$ be a C.O.N. set of functions of bounded variation, normalized so that $\alpha_{i}(1)=0$. Finally let

$$
\begin{aligned}
& \beta_{i}(t)=\int_{0}^{i} \alpha_{i}(s) d s \\
& c_{i}(x)=\int_{0}^{1} \alpha_{i}(s) d x(s) \quad\left(=-\int_{0}^{1} x(s) d \alpha_{i}(s)\right) .
\end{aligned}
$$

The question of the possibility of representation of $x \in C$ by

$$
x(t) \sim \sum_{i=1}^{\infty} c_{i}(x) \beta_{i}(t)
$$

where the right side of $(0.1)$ will be called the $c \beta$ expansion of $x$, arises in the following four ways.

(i) If $C$ is given the uniform norm, and if each $\beta_{i}^{\prime}(s)$ exists except for a finite number of points and is continuous except at these points and is bounded, then the set $\left\{\beta_{n}(t), c_{n}(x)\right\}$ is a biorthogonal system [1, p. 106] in terms of which the biorthogonal expansion of $x \in C$ is given by the right side of $(0.1)$. In this context then a relevant question is whether $(0.1)$ holds as an equality uniformly in $t$.

(ii) Paley, Wiener and Zygmund [12] have defined the P.W.Z. integral, denoted by

$$
\int_{0}^{1} f(s) \tilde{d} x(s)
$$

which, for any $f \in L_{2}(0,1)$, is defined for almost all $x \in C$ (in the sense of Wiener measure on $C)$. It will be shown in $\S 1$ that, for given $x \in C$ and given $f$ of bounded variation such that $f(1)=0$ (it will be seen that no loss of generality results from the assumption of this equality), the question of whether the P.W.Z. integral $\int_{0}^{1} f(s) \tilde{d} x(s)$, when it exists, agrees with the 
Riemann-Stieltjes integral $\int_{0}^{1} f(s) d x(s)$ is settled in the affirmative provided that suitable summability conditions of the $c \beta$ series of $x$ are satisfied. Theorem 1 of $\S 1$ specifies such conditions.

(iii) In the problem of approximating a Wiener integral

$$
\int_{c} F[x] d x
$$

it would be convenient to be able to approximate $x(s)$ by a sum

$$
x(s)=\sum_{k=1}^{n} b_{k}(s) c_{k}(x)
$$

for suitable functions $b_{k}(s)$. The reason is that then the simple integration formula [3, p. 115] for the Wiener integral of a functional $f\left(c_{1}(x), \ldots, c_{n}(x)\right)$ could be used. Routine formal computation from (0.2) suggests $b_{k}(s)=\beta_{k}(s)$. In this context convergence for almost all $x$ of the right side of $(0.1)$ to $x(s)$ in the uniform (or $L_{2}$ ) topology along with $F\left[\sum_{k=1}^{n} c_{k}(x) \beta_{k}(\cdot)\right]$ dominated by a Wiener integrable functional and $F$ continuous in the uniform (or $L_{2}$ ) topology would ensure that

$$
\lim _{n \rightarrow \infty} \int_{c} F\left[\sum_{k=1}^{n} c_{k}(x) \beta_{k}(\cdot)\right] d x=\int_{c} F[x(\cdot)] d x .
$$

(For results of this sort see [3], [5], [6], [9], [11].)

(iv) If $x^{\prime}(t)$ is continuous, indefinite integration of

$$
x^{\prime}(t)-\sum_{i=1}^{n} \int_{0} \alpha_{i}(s) x^{\prime}(s) d s \alpha_{i}(t)
$$

followed by an application of the Schwarz inequality shows that the $c \beta$ expansion of $x(t)$ converges uniformly to $x(t)$.

In this paper attention is focused on the problem as motivated in (ii) above by the P.W.Z. integral. As noted in (ii), Theorem 1 of $\S 1$ is then one of the important results. Theorem 2 specifies several C.O.N. sets of functions for which the conditions of Theorem 1 are satisfied for arbitrary $x \in C$. Also contained in $\S 1$ is an expansion theorem (Theorem 3 ) of arbitrary $x \in C$ in terms of the normalized characteristic functions of the Sturm-Liouville problem

$$
\left.\begin{array}{r}
y^{\prime \prime}(t)+\lambda w(t) y(t)=0, \\
y(0)=y^{\prime}(1)=0,
\end{array}\right\}
$$

where $w(s)>0$ and $w(s)$ has a continuous third derivative. The author believes this theorem is probably known but has been unable to find a reference for it. Thus an outline of the proof of this theorem is given as the content of $\S 3$. In $\S 4$ are given some concluding remarks about the $c \beta$ expansions considered in this paper. 
1. Agreement of $\int_{0}^{1} f(s) \tilde{d} x(s)$ with $\int_{0}^{1} f(s) d x(s)$.

The symbol $\int_{0}^{1} f(t) \alpha_{n}(t) d t$ will be replaced by $g_{n}$ below.

Definition. [12, p. 652]. For $f \in L_{2}(0,1)$ and $x \in C$ the limit

$$
\sum_{n=1}^{\infty} g_{n} \int_{0}^{1} \alpha_{n}(s) d x(s)
$$

if it exists, will be denoted by $\int_{0}^{1} f(s) \tilde{d} x(s)$ and called the P.W.Z. integral of $f$ with respect to $x$ (i.e. the $n$th approximation of $\int_{0}^{1} f(s) \tilde{d} x(s)$ is $\int_{0}^{1} f_{n}(s) d x(s)$, where $f_{n}(s)$ is the $n$th partial sum in the $\alpha$-expansion of $f$ ).

The principal theorems of the paper are the following.

THEOREM 1. Suppose that, for fixed $x \in C$, the following conditions are met:

(1) $\sum_{k=1}^{\infty} c_{k}(x) \beta_{k}(t)$ is summable by some permanent triangular series-to-sequence matrix method $A=\left(a_{m n}\right)$ to $x(t)$.

(2) The A-transform of $\sum_{k=1}^{\infty} c_{k}(x) \beta_{k}(t)$ is bounded in $t$ and $n$.

Then, for any $f$ of bounded variation, the series defining $\int_{0}^{1} f(t) \tilde{d} x(t)$ is A-summable to $\int_{0}^{1} f(t) d x(t)$ (which of course exists). Also, if $\int_{0}^{1} f(t) \tilde{d} x(t)$ exists, it equals $\int_{0}^{1} f(t) d x(t)$.

THEOREM 2. (i) The two conditions of Theorem 1 are satisfied, for all $x \in C$, by the following complete orthonormal sets of functions (normalized to be 0 at $t=1$ ):

(a) the sine functions,

(b) the cosine functions,

(c) the characteristic functions of (0.3),

(d) the Haar functions normalized to be right continuous.

(ii) Condition (1) of Theorem 1 is satisfied by

(e) the even Legendre polynomials.

The following theorem will be required in connection with (c) of Theorem 2.

THEOREM 3. The expansion of arbitrary $x \in C$ in the characteristic functions of $(0.3)$ is, for $\delta>0$, uniformly $(C, \delta)$ summable to $x$ on $[0,1]$.

Before a proof is given for Theorem 1 it will be noted in passing that Paley, Wiener and Zygmund [12] have shown that, for fixed $f \in L_{2}(0,1)$, the P.W.Z. integral exists for almost all $x \in C$ and that, for $f$ of bounded variation (in which case of course the R.S. integral exists for all $x \in C$ ), the two integrals agree for almost all $x$. 
The following lemma of Burkill [2, p. 74] will be required in the proof of Theorem 1.

LeMmA. Let $g(s)$ and $g_{n}(s)(n=1,2,3, \ldots)$ be continuous on $[0,1]$ and suppose that $\sum_{k=1}^{\infty} g_{k}(s)$ is summable by some permanent triangular series-to-sequence method $\left(a_{m n}\right)(m, n=$ $1,2,3, \ldots)$ to $g(s)$ on $[0,1]$. If the A-transform $G_{n}(s)=\sum_{k=1}^{n} a_{n k} g_{k}(s)$ converges boundedly to $g(s)$, then, for $h(s)$ of bounded variation,

(i) $\lim _{n \rightarrow \infty} \int_{0}^{1} G_{n}(s) d h(s)=\int_{0}^{1} g(s) d h(s)$,

(ii) If $\lim _{n \rightarrow \infty} \sum_{k=1}^{n} \int_{0}^{1} g_{k}(s) d h(s)$ exists, then this limit also equals $\int_{0}^{1} g(s) d h(s)$.

Proof. (i) appears in Burkill [2, p. 74]. The proof of (ii) follows from the fact that $\left(a_{m n}\right)$ to $g(s)$ is a permanent triangular matrix method. For then

$$
\int_{0}^{1} \sum_{k=1}^{n} g_{k}(s) d h(s) \rightarrow K
$$

implies that

$$
\sum_{k=1}^{n} a_{n k} \int_{0}^{1} g_{k}(s) d h(s) \rightarrow K
$$

or

$$
\int_{0}^{1} \sum_{k=1}^{n} a_{n k} g_{k}(s) d h(s)\left(=\int_{0}^{1} G_{n}(s) d h(s)\right) \rightarrow K .
$$

From (i) it follows that $K=\int_{0}^{1} g(s) d h(s)$ and the proof of (ii) is complete.

Next follows the proof of Theorem 1. It is first noted that $f(1)=0$ can (and will) be assumed so far as the existence of, or the value of, either $\int_{0}^{1} f(s) \tilde{d} x(s)$ or $\int_{0}^{1} f(s) d x(s)$ is concerned. Now, for $f$ of bounded variation (recall the definition of $g_{n}$ at the beginning of this section),

$$
\begin{aligned}
g_{k} & =\left.f(t) \int_{0}^{t} \alpha_{k}(s) d s\right|_{0} ^{1}-\int_{0}^{1} \int_{0}^{t} \alpha_{k}(s) d s d f(t) \\
& \left.=-\int_{0}^{1} \int_{0}^{t} \alpha_{k}(s) d s d f(t) \quad \text { (because } f(1)=0\right) \\
& =-\int_{0}^{1} \beta_{k}(t) d f(t) .
\end{aligned}
$$


Thus

$$
\begin{aligned}
& \int_{0}^{1}\left[\sum_{k=1}^{n} g_{k} \alpha_{k}(s)\right] d x(s) \\
& \quad=\left.\sum_{k=1}^{n} g_{k} \alpha_{k}(s) x(s)\right|_{0} ^{1}-\int_{0}^{1} x(s) d\left[\sum_{k=1}^{n} g_{k} \alpha_{k}(s)\right] \\
& =-\int_{0}^{1} x(s) d\left[\sum_{k=1}^{n} g_{k} \alpha_{k}(s)\right] \quad\left(\text { since } \alpha_{k}(1)=x(0)=0\right) \\
& \left.=\int_{0}^{1} \sum_{k=1}^{n} \int_{0}^{1} x(s) d \alpha_{k}(s) \beta_{k}(t) d f(t) \quad \text { (because of }(1.1)\right) \\
& =-\int_{0}^{1} \sum_{k=1}^{n} c_{k}(x) \beta_{k}(t) d f(t) .
\end{aligned}
$$

Also

$$
\int_{0}^{1} f(t) d x(t)=-\int_{0}^{1} x(t) d f(t)
$$

It follows from (1.2) and (1.3) and the lemma that the series defining the P.W.Z. integral is $A$-summable to the R.S. integral and that, if this series converges, it equals $\int_{0}^{1} f(t) d x(t)$. The proof is complete.

2. Convergence behaviour for specific $\alpha$-sets. The proof of Theorem 2 will now be given. It is important to note that $\alpha(1)=0$. Furthermore, each of the $\alpha$-sets investigated, except the Haar set, has each $\alpha$ absolutely continuous on $[0,1)$ and so in these cases

$$
\sum_{k=1}^{n} c_{k}(x) \beta_{k}(t)=-\sum_{k=1}^{n} \int_{0}^{1} x(s) \alpha_{k}{ }^{\prime}(s) d s \beta_{k}(t)+\sum_{k=1}^{n} x(1) \alpha_{k}(1-) \beta_{k}(t)
$$

(where $\alpha_{k}(1-)$ is the limit from the left). Now follows the convergence investigation for the special cases mentioned.

(a) Sines: $\alpha_{k}(s)=\sqrt{ } 2 \sin k \pi s$.

$$
\sum_{k=1}^{n} c_{k}(x) \beta_{k}(t)=\sum_{k=1}^{n}\left[\int_{0}^{1} x(s) \sqrt{2} \cos k \pi s d s\right] \sqrt{ } 2 \cos k \pi t-\int_{0}^{1} x(s) \sum_{k=1}^{n} 2 \cos k \pi s d s
$$

By a standard theorem in Fourier series the first member in the right side of (2.2) is, for $\delta>0$, uniformly $(C, \delta)$ summable to $x(t)-\int_{0}^{1} x(s) d s$ on $[0,1]$ and the second member is uniformly $(C, \delta)$ summable to $-x(0)+\int_{0}^{1} x(s) d s$, so that the left side is uniformly $(C, \delta)$ summable to $x(t)$ on $[0,1]$. The conditions of Theorem 1 are thus clearly satisfied.

$c$ 
(b) Cosines: $1, \sqrt{2} \cos \pi s, \sqrt{ } 2 \cos 2 \pi s, \ldots$ Here

$$
\begin{aligned}
\sum_{k=1}^{n+1} c_{k}(x) \beta_{k}(t)= & x(1) t+\sum_{k=1}^{n} \int_{0}^{1} x(s) \sqrt{2} \sin k \pi s d s \sqrt{2} \sin k \pi t \\
& +x(1) 2 \sum_{k=1}^{n}(-1)^{k} \sin k \pi t / k \pi .
\end{aligned}
$$

The sum of the two sums on the right of (2.3) is the $n$th partial sum of the Fourier series expansion of $x(t)-x(1) t$ on $[0,1]$ and so, by a standard theorem, is uniformly $(C, \delta)$ summable on $[0,1]$ to $x(t)-x(1) t$. Again then the conditions of Theorem 1 are satisfied.

(c) The normalized characteristic functions of the Sturm-Liouville problem:

$$
\left.\begin{array}{c}
\left\{[1 / w(s)] \alpha^{\prime}(s)\right\}^{\prime}+\lambda \alpha(s)=0, \\
\alpha^{\prime}(0)=\alpha(1)=0 \text { on }[0,1],
\end{array}\right\}
$$

where $w(s)>0$ and $w(s)$ has a continuous third derivative.

In this case

$$
-\int_{0}^{1} x(s) \alpha_{k}^{\prime}(s) d s=\int_{0}^{1} x(s) \lambda_{k} w(s) \beta_{k}(s) d s,
$$

where the $\lambda$ 's are the characteristic numbers of (2.4) (because $\alpha_{k}^{\prime}(s)=-\lambda_{k} w(s) \beta_{k}(s)$ ) and thus

$$
\sum_{k=1}^{n} c_{k}(x) \beta_{k}(t)=\{1 /[w(t)]\} \sum_{k=1}^{n}\left[\int_{0}^{1}\left\{x(s) \sqrt{ }[w(s)] \sqrt{ }\left[\lambda_{k} w(s)\right] \beta_{k}(s) d s \sqrt{ }\left[\lambda_{k} w(t)\right] \beta_{k}(t)\right\}\right] .
$$

Now $\left\{\sqrt{ }\left[\lambda_{n} w(t)\right] \beta_{n}(t)\right\}$ is known to be C.O.N. [5, p. 463]. In fact $\left\{\beta_{n}(t)\right\}$ can easily be shown to be the set of characteristic functions of the S-L problem (0.3); this set is orthogonal with respect to the weight $w(t)$. Since $x(t) \sqrt{ }[w(t)] \in C$, Theorem 3 shows that the right side of (2.5) is uniformly Cesàro summable to $x(t)$ and again the conditions of Theorem 1 have been shown to be satisfied.

(d) The Haar functions normalized to be right continuous. Ciesielski [4] has shown that for each $x \in C$ the graph of $\sum_{k=1}^{n+1} c_{k}(x) \beta_{k}(t)$ has at least the same vertices as that of $\sum_{k=1}^{n} c_{k}(x) \beta_{k}(t)$ and that $\left\{\sum_{k=1}^{n} c_{k}(x) \beta_{k}(t)\right\}$ converges uniformly to $x(t)$. An outline of a proof of this is given in [6]. In this case then the conditions of Theorem 1 are of course satisfied and in fact for every $x \in C$ and every $f$ of bounded variation (with $f(1)=0) \int_{0}^{1} f(t) \tilde{d} x(t)$ exists and equals $\int_{0}^{1} f(t) d x(t)$

(e) The even Legendre polynomials on $[0,1]$ :

$$
\alpha_{2 k}(s)=\sqrt{ }(4 k+1) P_{2 k}(s) \quad(k=0,1,2, \ldots) .
$$


(cf. [10, (4.7.15), p. 81] and note that the $n$th Legendre polynomial $P_{n}(s)$ is there given as the $n$th ultraspherical polynomial $\left.P_{n}^{(1 / 2)}(s)\right)$,

$$
\beta_{2 k}(t)=\left\{\begin{array}{l}
t \quad(k=0), \\
\sqrt{ }(4 k+1)\left(t^{2}-1\right) P_{2 k}^{\prime}(t) /[2 k(2 k+1)] \quad(k \geqq 1),
\end{array}\right\}
$$

(because $\left[\left(u^{2}-1\right) P_{2 k}^{\prime}(u)\right]^{\prime}=2 k(2 k+1) P_{2 k}(u)[10,(4.7 .5)]$ and because $P_{2 k}^{\prime}(0)=0[10,(4.7 .14)$ and (4.7.4)]).

Now

$$
\left.\begin{array}{l}
P_{0}{ }^{\prime}(t)=0, \\
P_{2 k}^{\prime}(t)=P_{2 k-1}^{(3 / 2)}(t) \quad(k \geqq 1)[9,(4.7 .14)],
\end{array}\right\}
$$

and it is easy to verify $[10,(4.7 .15)$ and (4.7.4)] that the set

$$
\left\{\gamma_{2 k}(t)=\sqrt{ }\left[\left(1-t^{2}\right)(4 k+1)\right] P_{2 k-1}^{(3 / 2)}(t) / \sqrt{ }[2 k(2 k+1)]: k \geqq 1\right\}
$$

is C.O.N. on $[0,1]$. Also the first sum on the right of $(2.1)$ is easily verified by (2.6) and (2.7) to be

$$
\sqrt{ }\left(1-t^{2}\right) \sum_{k=1}^{n} \int_{0}^{1}\left[x(s) / \sqrt{ }\left(1-s^{2}\right)\right] \gamma_{2 k}(s) d s \gamma_{2 k}(t),
$$

i.e. $\sqrt{ }\left(1-t^{2}\right)$ times the $n$th partial sum in a Jacobi expansion of $x(t) / \sqrt{ }\left(1-t^{2}\right)$. The second sum on the right of $(2.1)$ is

$$
x(1) t+x(1) \sum_{k=1}^{n}\left[P_{2 k+1}(t)-P_{2 k-1}(t)\right]
$$

(which follows easily from

$$
\begin{aligned}
& \beta_{2 k}(t)=\sqrt{ }(4 k+1) \int_{0}^{t} P_{2 k}(s) d s \text { and } \\
& P_{2 k}(u)=\left[P_{2 k+1}^{\prime}(u)-P_{2 k-1}^{\prime}(u)\right] /(4 k+1) \quad[10,(4.7 .29)]
\end{aligned}
$$

along with $\left.P_{2 k-1}(0)=0\right)$. Now (2.9) converges to 0 on $[0,1)$ because of the telescoping property, the estimate [10, (7.3.8), p. 160] for $P_{n}(t)$, and the fact that $P_{1}(t)=1$. At 1, (2.9) converges to $x(1)$ because $P_{2 k+1}(1)=0$. Also, it is easy to see that the convergence is bounded on $[0,1]$ and uniform on $[0,1-\varepsilon]$. By a theorem of Szego [10, Theorem 9.1.2, p. 239] the expression (2.8) is, for $\delta>0$, summable $(C, \delta)$ uniformly on $[0,1-\varepsilon]$ to $x(t)$ and clearly (2.8) converges to 0 at $t=1$ (there it is always 0 ). Thus the $c \beta$ series satisfies condition (1) of Theorem 1. The author has been unable to find whether condition (2) is also satisfied. What is required is to show that, for suitable $M>0, M / \sqrt{ }\left(1-t^{2}\right)$ is a majorant for the Cesàro approximations in the Jacobi expansion of $x(t) / \sqrt{ }\left(1-t^{2}\right)$. That there is such a majorant for the partial sums of an ordinary Fourier series can be shown from the results of Zygmund [13, p. 154] but a similar technique for Jacobi series does not seem to follow easily. 
3. Theorem 3. An outline of the proof of Theorem 3 will now be given. First one verifies that for $w(s) \equiv 1$ there results for the characteristic functions of $(0.3)$

$$
y_{k}(t)=\sqrt{ } 2 \sin [(2 k-1) \pi t / 2] \text {. }
$$

Then it is easy to verify that, for $x \in C$, the ordinary Fourier sine expansion of $z(t)$, defined by

is

$$
z(t)=\left\{\begin{array}{l}
x(t) \text { for } t \in[0,1] \\
x(2-t) \text { for } t \in[1,2]
\end{array}\right.
$$

$$
\sum_{k=1}^{n}\left[\left((-1)^{k+1}+1\right) / 2\right] \int_{0}^{1} x(s) \sqrt{2}[\sin k \pi s / 2] d s \cdot \sqrt{ } 2 \sin k \pi t / 2 .
$$

(the inclusion of the zero terms in (3.1) is important in the context of summability below). The theory of Cesàro summability for Fourier series ensures that the series for which (3.1) is the $n$th partial sum is uniformly summable $(C, 1)$ to $z(t)$ on the real line. Thus the $y_{k}$ expansion of $x(s)$ on $[0,1]$, the $n$th partial sum of which is also computed to be (3.1) with the zero terms deleted, is uniformly summable to $x(t)(=z(t)$ on $[0,1])$ by the permanent triangular method whose matrix is that of $(C, 1)$ means with alternate columns deleted.

In fact, the series having the $n$th partial sum of (3.1) with the zeros deleted as its $n$th partial sum is uniformly $(C, \delta)$ summable for $\delta>0$. This can be proved as follows from the equivalence, for $\delta>0$, of Cesàro summability $(C, \delta)$ and Riesz summability $(R, n, \delta)$. Let the series corresponding to (3.1) with alternate zero terms deleted and the series corresponding to (3.1) as it stands be denoted respectively by

where

$$
\begin{aligned}
& a_{1}(t)+a_{2}(t)+a_{3}(t)+\ldots, \\
& b_{1}(t)+b_{2}(t)+b_{3}(t)+\ldots,
\end{aligned}
$$

$$
\left.\begin{array}{c}
b_{2 n-1}(t)=a_{n}(t) \\
b_{2 n}(t)=0 .
\end{array}\right\}
$$

The $(R, n, \delta)$ summability of (3.2) or (3.3) is defined as meaning respectively that $\sigma_{1}(u, t) \rightarrow f(t)$ or $\sigma_{2}(u, t) \rightarrow f(t)$ as $u \rightarrow \infty$, where

By (3.4),

$$
\begin{aligned}
& \sigma_{1}(u, t)=u^{-\delta} \sum_{n<u}(u-n)^{\delta} a_{n}(t), \\
& \sigma_{2}(u, t)=u^{-\delta} \sum_{n<u}(u-n)^{\delta} b_{n}(t) .
\end{aligned}
$$

$$
\begin{aligned}
\sigma_{2}(u, t) & =u^{-\delta} \sum_{2 n-1<u}(u-(2 n-1))^{\delta} a_{n}(t) \\
& =2^{\delta} u^{-\delta} \sum_{n<(u+1) / 2}[(u+1) / 2-n]^{\delta} a_{n}(t) \\
& =[(u+1) / u]^{\delta} \sigma_{1}[(u+1) / 2, t] .
\end{aligned}
$$

Thus $\sigma_{2}(u, t) \rightarrow f(t)$ uniformly as $u \rightarrow \infty$ if and only if $\sigma_{1}(u, t) \rightarrow f(t)$ uniformly as $u \rightarrow \infty$. 
The proof of the theorem for $w(s) \equiv 1$ is complete.

Next, for general $w(s)$, the expansion result is obtained in terms of the characteristic functions of the Liouville normal form of $(0.3)$ by means of the estimate in Kamke [8, p. 263] for the characteristic functions and the expansion theorem [8, p. 261], along with the technique given by Ince [7, pp. 276-278]. Finally, the expansion is obtained in terms of the characteristic functions of $(0.3)$ (not in normal form) by the observation that the expansion of $x(t)$ in characteristic functions of $(0.3)$ is $\{w[t(s)]\}^{1 / 4}$ times the expansion of $x[t(s)] /\{w[t(s)]\}^{1 / 4}$ in characteristic functions of the normal form of (0.3), where $s=\int_{0}^{t} \sqrt{ }[w(v)] d v / K$ as used in the Liouville transformation. The outline of the proof is complete.

4. Concluding remarks. It will be noted that in examples (a)-(c) convergence properties of the $c \beta$ series were obtained from the fact that the first sum on the right of (2.1) is a partial sum in an orthonormal expansion. This in turn was due to the fact that the $\alpha$ 's were solutions of a Sturm-Liouville problem for which the differential equation was of the form

$$
\left[r(x) y^{\prime}\right]^{\prime}+[p(x)+\lambda g(x)] y=0,
$$

where $p \equiv 0$ and $g(x)$ is a constant $M$. This results in

$$
\int_{0}^{x} y(u) d u=r(x) y^{\prime}(x) / \lambda M
$$

and so makes the $\beta$ 's be of the form (const.) $\left(r(x) \alpha^{\prime}(x)\right)$; hence the summands in the first term of the right side of (2.1) are then at least of the form of summands in an O.N. expansion.

The Legendre example is of interest in that only for that case is the differential equation singular.

The Haar functions, which yield $\beta$ 's having no nice orthogonality properties, give the best $c \beta$ series of those considered.

It would be interesting to find a technique for investigating the $c \beta$ series convergence properties of more general Sturm-Liouville problems-perhaps the ultrasphericals for example. The author has been unable to do so.

The author thanks the referee for several corrections and helpful suggestions.

\section{REFERENCES}

1. Stefan Banach, Théorie des opérations linéaires (Warsaw, 1932).

2. J. C. Burkill, The Lebesgue integral, Cambridge Tract No. 40 (Cambridge, 1961).

3. R. H. Cameron, A "Simpson's Rule" for the numerical evaluation of Wiener's integrals in function space, Duke Math. J. 18 (1951), 111-130.

4. Z. Ciesielski, On Haar functions and on the Schauder basis of the space $C_{(0,1) \text {. (Russian }}$ summary, unbound insert), Bull. Acad. Polon. Sci. Sér. Sci. Math. Astronom. Phys. 7 (1959), 227-232, Math. Reviews 24A, 1599.

5. H. C. Finlayson, Vladimirov-type approximation of Wiener integrals of suitably dominated functionals, Trans. Amer. Math. Soc. 132, 2 (1968), 461-469.

6. H. C. Finlayson, Approximation of Wiener integrals of functionals continuous in the uniform topology, Pacific J. Math. 34 (1970), 61-71. 
7. E. L. Ince, Ordinary differential equations (New York, 1956).

8. E. Kamke, Differentialgleichungen Lösungsmethoden und Lösungen, 3rd edn. (New York, 1948).

9. Alan G. Konheim and Willard L. Miranker, Numerical evaluation of Wiener integrals, Math. Comp. 21 (1967), 49-65.

10. G. Szego, Orthogonal polynomials, Amer. Math. Soc. Colloquium Publications, Vol. 23 (New York, 1939).

11. V. S. Vladimirov, The approximate evaluation of Wiener integrals, Uspeki Mat. Nauk 15 (1960), 4 (94), 129-135. English translation: Amer. Math. Soc. Transl. (2) 34 (1963), 405-412.

12. R. E. A. C. Paley, N. Wiener and A. Zygmund, Notes on random functions, Math. Zeit. 37 (1933), 647-668.

13. A. Zygmund, Trigonometric series, vol. I, 2nd edn. (Cambridge, 1959).

UNIVERSITY OF MANITOBA

Manitoba, Canada 